\title{
¿Por qué ven Netflix quienes ven Netflix?: experiencias de engagement de jóvenes mexicanos frente a quien revolucionó el consumo audiovisual
}

\author{
Why do those who watch Netflix watch Netflix? Engagement \\ experiences of young Mexicans facing the one who \\ revolutionized audiovisual consumption
}

\author{
Benavides Almarza, C. F. y García-Béjar, L. ${ }^{1}$ \\ Recibido: 14-08-2020 - Aceptado: 8-02-2021 \\ https://doi.org/10.26441/RC20.1-2021-A2
}

RESUMEN: La masificación de plataformas de streaming, específicamente Netflix, ha modificado las formas de consumo de una audiencia cada vez más activa y empoderada a la hora de elegir cómo, cuándo y qué contenido ver. Este patrón de comportamiento ha generado que se exploren nuevos mecanismos para conocer con mayor detalle y profundidad las motivaciones y emociones que tienen las personas cuando se enfrentan a contenidos y plataformas audiovisuales.

La medición del engagement ofrece información adecuada para predecir esos comportamientos que condicionan el consumo, así como algunas características que provocan fidelidad hacia una marca y la disposición por un servicio. Este trabajo describe, a través de un análisis cuantitativo factorial, las experiencias de engagement que 574 millennials mexicanos tienen al usar Netflix. Se logró establecer que al usar Netflix sobresalen experiencias relacionadas con la socialización, entretenimiento y reflexión frente a nuevos temas. Lo que indicaría que más allá del ocio, genera otro tipo de atributos a los que típicamente se le asocian.

Palabras clave: engagement; jóvenes mexicanos; plataformas de streaming; Netflix; México; audiencia.

\begin{abstract}
The massification of streaming platforms, specifically Netflix, has modified the consumption patterns of an increasingly active and empowered audience when it comes to choosing how, when and what content to watch. This behavioral pattern has generated the exploration of new mechanisms to know in greater detail and depth the motivations and emotions that people have when they face audiovisual content and platforms.

The measurement of engagement offers adequate information to predict those behaviors that condition consumption, as well as some characteristics that cause loyalty to a brand and willingness to pay for a service. This paper describes, through a quantitative factor analysis, the engagement experiences that 574 Mexican millennials have when using Netflix. It was established that when using Netflix, experiences related to socialization, entertainment and reflection on new topics stand out. This would indicate that beyond entertainment, it generates other types of attributes that are typically associated with it.
\end{abstract}

Keywords: engagement; young mexicans; streaming platforms; Netflix; México; audience.

\footnotetext{
${ }^{1}$ Cristóbal Fernando Benavides Almarza es doctor en Comunicación Pública por la Universidad de Navarra y Decano de la Facultad de Comunicación de la Universidad de los Andes (Chile). cbenavides@uandes.cl, https:// orcid.org/0000-0001-5573-6785

Ligia García-Béjar es estudiante del Doctorado de la Universidad de los Andes (Chile), magister en Comunicación por la misma institución y Secretaria de Investigación de la Escuela de Pedagogía en la Universidad Panamericana, Campus, Guadalajara (México). ligarcia@up.edu.mx, https://orcid.org/0000-0002-2980-0314
} 


\section{Introducción}

Una persona cualquiera ha pasado muchas horas de su vida consumiendo contenidos audiovisuales y el 2020, año de pandemia, ha confirmado aún más esa tendencia. Los mexicanos pasaron en promedio el año pasado más horas frente a las pantallas. El tiempo de exposición a la televisión abierta aumentó en 13 minutos, en la de pago 9 minutos y otras plataformas 17 minutos (Nielsen, 2020).

Pero esos no han sido los únicos cambios. La industria audiovisual está en medio de una revolución, en la que se ha pasado de un paradigma de la distribución a un paradigma del consumo, donde las decisiones principales están en manos del consumidor, "una vez que el contenido se digitaliza, controlar su distribución se convierte en una tarea sumamente compleja en la que el poder y la logística parecen estar en manos del usuario" (De la Gala, 2016, p. 39).

La libertad de consumo del usuario también ha generado que la lealtad hacia determinadas marcas y medios sea cada vez más frágil (Eizaguirre \& Peñafiel, 2013; Bergillos, 2017), complicando la situación económica de muchas de ellas. Las empresas de medios han tenido que reaccionar para frenar la caída de ingresos y enfrentar la hiperfragmentación de la audiencia (Barthel, 2019). Para encarar este problema algunas han optado por generar nuevas alianzas, mientras que otras por ampliar las plataformas (Newman, Fletcher, Levy \& Kleis Nielsen, 2019) por las cuales se pueden conectar de manera más eficiente con su audiencia y anunciantes (García, Salaverría y Masip, 2008).

Para Medina, Herrero y Urgellés (2017) esta nueva realidad ha supuesto la aparición de nuevos competidores con tecnologías capaces de responder a las necesidades e intereses de diferentes tipos de usuarios. En este ecosistema, las empresas de comunicación existentes deben ser lo suficientemente flexibles para sortear a los nuevos competidores, detectar las oportunidades e identificar fortalezas en un escenario en el que las audiencias buscan contenidos accesibles, atractivos y accesibles en términos monetarios (Neira, 2015).

Entre las opciones tecnológicas que han surgido destacan las plataformas de streaming bajo demanda (VoD) o de transmisión de contenido a través de internet, con sistemas de suscripción pagada o gratuita, y otras simplemente de libre acceso. Para Lotz (2014) el valor que han traído estas compañías (Netflix, Amazon Prime, Disney+ y Apple TV, entre otras) es la concentración de su estrategia en el consumidor, más que en el anunciante. Sus modelos de negocios e ingresos se basan mayoritariamente en el pago que mensualmente realizan sus suscriptores.

A eso hay que sumarle el amplio conocimiento del comportamiento de sus públicos gracias a los algoritmos y uso de inteligencia artificial que les permiten obtener datos sobre lo que ven y de esa manera darles recomendaciones y mantenerlos interesados en sus contenidos (Jenner, 2017). No obstante esta información es sumamente relevante para la definición de sus estrategias de distribución y producción de contenidos; la data sobre los usuarios abre un espacio para profundizar más sobre las experiencias y los vínculos que los usuarios desarrollan al consumir estas opciones de consumo. En este sentido, esta investigación busca aportar información sobre diversas experiencias de engagement que los millennials del área metropolitana de Guadalajara (México) tienen con Netflix (principal plataforma de consumo audiovisual de streaming en el país), en función del tiempo y la forma en la que sucede ese consumo.

La evolución de las audiencias exige una evolución en la medición, aproximación y estudio de ellas. "En la medida de que las pantallas tradicionales ya no tienen un rol predominante en la vida de las personas. La flexibilización de los procesos en los que la industria genera contenidos impacta en la manera en la que se lleva a cabo la vinculación con los públicos, como señalan Papí-Galvez \& Perlado-Lamo" (2018, p. 384). Además, en un entorno multimedia, el público es consumidor de contenidos, es usuario de tecnología (Ferrucci, 2017) y también prosumidor. Esta condición poli- 
sémica de las audiencias reclama la necesidad de encontrar diversas formas de entender el consumo de un público conectado permanentemente y que obtiene satisfactores distintos de los productos de comunicación que busca. Para Havens \& Lotz (2016), los cambios más notables son las opciones de consumo a disposición del público, mediadas por los algoritmos de recomendación, la fragmentación producto de la producción individualizada de opciones y la conveniencia, entendida como la facilidad para que las personas consuman e interactúen.

\subsection{La industria de contenidos en México y sus audiencias}

El modelo de negocio de la industria audiovisual mexicana tradicionalmente se ha sostenido gracias a la publicidad, permitiendo que el público pudiera consumir medios de comunicación sin pagar nada. Este esquema se rompió paulatinamente primero con la irrupción de los sistemas de cable y luego con las OTT. En ambos casos se pudo comprobar que el público estaba dispuesto a pagar por un servicio que le agregara valor (Gutiérrez-Rentería, 2014).

Según los datos de la Asociación de Internet en México (2020), el 75.8\% de la población accede a internet y más del $50 \%$ de esta cifra corresponde a personas entre los 18 y 35 años, pasando un promedio de 8 horas y 20 minutos de su día usando internet para algún propósito. Además, el 57\% de esos usuarios utiliza internet varias veces a la semana para ver películas o series en alguna plataforma de streaming (IFT, 2019). El internauta mexicano tiene en promedio 4.91 dispositivos en su hogar (teléfono celular, computadora portátil, televisión inteligente, tableta y consola de videojuegos) y se conecta a internet con 3.62 de éstos (IAB, 2020).

Las previsiones del mercado sugieren que la emergencia sanitaria por la Covid19 impulsará un crecimiento de $26 \%$ en los ingresos de las plataformas de video streaming como Netflix, Claro Video y Amazon Prime para el cierre de este año (PwC, 2020). Con información de Global Entertainment and Media Outlook 2020-2024 de PwC, Statista (2020) e IAB (2020), el 89\% de los usuarios de internet en México declaran tener acceso a servicios de entretenimiento de paga por internet, de lo cuales, $82 \%$ son a streaming de video, $59 \%$ a streaming de audio y $21 \%$ a streaming de videojuegos. Las OTT en México tienen un crecimiento mayor que a nivel global y en toda Latinoamérica, del 2017 al 2018 crecieron a un porcentaje del 20.6\%. A nivel global y Latinoamérica el crecimiento es de $10 \%$ y $11 \%$, respectivamente. Dicho aumento en la penetración de mercado supone ganancias en promedio de 83 billones de dólares para las empresas de OTT a nivel mundial y la expectativa es que para 2024, éstas serán de 158 billones de dólares.

En 2020, de acuerdo a Statista (2020) e IFT (2019) México representó 1,038 millones de dólares de ingresos para estas compañías, un alza del 26\% respecto al 2019. Del 2016 al 2019 el incremento de usuarios de plataformas pagadas subió del $62 \%$ al $77 \%$ y los formatos más consumidos fueron: películas (84\%), series (74\%), documentales (34\%) y contenidos deportivos (21\%). Hacia finales del 2018, el ranking de compañías en México de acuerdo a la cantidad de usuarios lo encabeza Netflix con una penetración de mercado de 50\%, seguido por Claro Video (15\%), Amazon Video (10\%) Blim (5\%), YouTube Red (5\%), Crunchyroll (5\%) y HBO Go, Apple TV entre otros (10\%).

En 2017, de acuerdo a un reporte de Netflix, México fue el país de Latinoamérica con más horas de consumo al día, con un promedio de 9.5 horas de consumo y una media de visualización de 60 películas al año (Netflix Media Center, 2017). El promedio llegó a 9.5 horas y con una media de 60 películas al año. Así mismo, los datos más recientes de suscripción a esta SVOD reportaron 25.9 millones de usuarios en el país al 2020 (Statista, 2020b), con un alcance promedio de 74.3 millones de usuarios (Nielsen-IBOPE, 2020). Una de las estrategias de Netflix en el mercado mexicano ha sido la producción de contenido original regional. En el 2017, el servicio reportó que tan solo en Latinoamérica tenía 50 distintas producciones en diversas etapas de desarrollo en Argentina, Brasil, Chile, Colombia 
y México. Las primeras series originales que produjo la compañía en México fueron el "Club de Cuervos", seguida por "Ingobernable", "El Chapo", "Luis Miguel: la serie", "La Casa de las Flores" y "Made in México", además de varios especiales de comedia (Netflix Media Center, 2017). En los últimos dos años, esta compañía ha realizado más de 50 proyectos entre series, películas y documentales, para el 2020 tiene dedicada una inversión de 200 millones de dólares en contenidos regionales (Armendáriz, 2019) y para el 2021, de 300 millones (Hernández y Aguilar, 2021).

A partir de los datos anteriores, se observa la pertinencia de analizar con más detalle a la audiencia cautiva de Netflix en México. La naturaleza impredecible de las audiencias motiva a la industria a desarrollar variedad de técnicas y maneras de predecir y conocer el comportamiento del público. Esto con el objeto de reducir el riesgo al momento de producir un contenido de comunicación y mejorar los canales de distribución y producción de un producto. En un mercado de contenidos caracterizado por la socialización del consumo y la fragmentación, se vuelve indispensable comprender mejor a las audiencias y lo que implica consumir contenidos en un mercado audiovisual convergente. Tradicionalmente, las audiencias eran segmentadas según su edad, género, lugar de residencia, estrato socioeconómico, nivel educativo (Orozco, 1997). Sin embargo, en los últimos años ha aparecido una nueva tendencia que busca comprender con mayor detalle las motivaciones, sentimientos y sensaciones del por qué una persona consume un determinado producto y/o servicio.

\section{Engagement en el consumo de medios digitales}

En el ámbito de la comunicación, los modelos de engagement han sido ampliamente discutidos desde la academia y la industria, $y$, al mismo tiempo, ha sido complejo definirlo de manera unitaria. En los últimos años, la importancia de analizar el comportamiento de los diferentes públicos ha ido en aumento, tomando relevancia el concepto de engagement de la audiencia (Ferrer-Conill, \& Tandoc, 2018).

"El término de engagement pone de manifiesto las dimensiones psicológicas y de comportamiento que explican la vinculación a nivel individual y social de una persona en relación con algo más" (Johntson y Taylor, 2018, p. 4). Las distintas perspectivas disciplinarias sobre el engagement lo sitúan como una entidad binaria que explica la riqueza interrelacional y el significado compartido entre una persona y un objeto (entre el consumidor y una marca; entre un estudiante y un maestro; entre un ciudadano y el sistema político, entre un empleado y la empresa, etc.), con influencia de variables personales y el contexto social (Barry y Crant, 2000). "El engagement se define como un concepto relacional, dinámico y multidimensional que incluye atributos psicológicos y conductuales de conexión, interacción, participación e involucramiento, diseñados para lograr u obtener un resultado a nivel individual, organizacional o social" (Johntson y Taylor, 2018, p. 19).

Las primeras aplicaciones en la industria de la comunicación del engagement tienen su origen en la publicidad y el marketing (Calder, Malthouse \& Schandel, 2009; Mollen \& Wilson, 2010 \& Hollebeck, 2011) y al usarlo se pretendía entender el nivel de implicación y motivación que tienen los usuarios de un servicio, así como el proceso psicológico que implica a una persona en su consumo (Bowden, 2009). Una aproximación desde la experiencia de consumo mediático y con origen en la experiencia de marca, es la de Higgins \& Scholer (2009) que hacen referencia a un estado de involucramiento y de atención profunda hacia un algo. En esa misma línea Calder (2010) habla de este como una serie de experiencias multinivel que emergen de las motivaciones, los pensamientos y los sentimientos que las personas tienen cuando experimentan con un consumo.

Para Napoli (2010) la transformación de la industria de lo tradicional a lo digital, ha posibilitado en particular, la creación de modelos de medición de audiencias, como los relativos a la medición de vínculos que se producen con determinados contenidos. Es en esa dimensión, en la que la medición 
del engagement ha ido ganando adeptos como una buena opción para conocer desde una dimensión más completa el comportamiento de audiencias que son más interactivas y participativas (Van Weezel \& Benavides, 2013). Esto no sólo con el objetivo de conocer sus gustos, preferencias y motivaciones, sino que también poder monetizar su comportamiento dada la vinculación emocional, sentimental y activa de ellas (Napoli, 2010).

Existe a su vez el engagement platform, que supone la relevancia de las interfaces como facilitador de la construcción del vínculo desarrollado por un usuario hacia una marca, producto o servicio (Brodie, Ilić, Jurić \& Hollebeek, 2013; Harris \& Dennis, 2011). Las plataformas que facilitan la interacción de los usuarios son las que suelen alcanzar mayores niveles de engagement (Van Weezel \& Benavides, 2010). En esa misma línea Lin \& Parsons (2018) argumentan que el engagement puede ser útil a la hora de que los usuarios se mantengan conectados a una plataforma o contenido cuando están realizando multitareas.

Chan-Olmsted \& Wolter (2018) propusieron una definición de engagement como el compromiso de una construcción multidimensional, que posibilita ahondar en detalles más complejos respecto de los estudios tradicionales; a través del engagement se logran describir componentes clave de la relación del público con los productos que consume. Moe, Poell \& Van Dijck, (2016) visualizaban la importancia de medir el engagement de las audiencias, como una alternativa complementaria al rating, que se limitaba a dar información sobre la exposición a un producto, pero carecía de elementos para entender las dinámicas de un público que interactúa y se vincula personalmente con los productos que consume, usando las redes sociales, por ejemplo. Mientras que Belair-Gagnon, Nelson \& Lewis (2019) concluyen que el engagement debe ser impulsado por los mismos creadores de contenido (periodistas, guionistas, productores) ya que en la medida que ellos privilegian la participación y relación más allá de sus tareas cotidianas, pueden generar mayor confianza y fortalecer los lazos con su público. Para eso se deben dedicar horas y procesos formales al interior de la organización, de modo de implementarlo de manera ordenada y, sobretodo, productiva (Neilson, 2018).

Una de las principales razones por las cuales el engagement fue propuesto como indicador de desempeño en la industria de medios fue la limitación en la entrega de información de los procesos de medición del rating en la profundización sobre el conocimiento de las personas inmersas en el consumo de un contenido (González-Bernal, 2016). Para Napoli (2011) el consumo de medios pasa por distintas dimensiones que van desde la conciencia, el anhelo o la necesidad de establecer relaciones con algún medio o contenido. Mientras que Oeldorf-Hirsch (2018) se aproxima al engagement como la capacidad de involucrar a los usuarios en el acceso al contenido, siendo las redes sociales una buena vía para generarlo.

Denktaş-Şakar, \& Sürücü (2018) consideran que el engagement es una herramienta fundamental a la hora de medir los niveles de participación de la audiencia y los públicos relacionados. Datos que permiten gestionar estrategias para construir relaciones fructíferas y duraderas. Cuando se trata de jóvenes puede ser un poco más complejo, porque los jóvenes millennials se caracterizan por estar en constante cambio (incluido su comportamiento frente a medios y contenido). Hermans, \& Prins (2020) sugieren que para fomentar el engagement de los jóvenes, una de las mejores estrategias es ser constructivos en esa relación y vincular el contenido que se les ofrece con los aspectos relevantes de sus vidas. Gulyas, O'Hara \& Eilenberg (2019) se aproximan al engagement desde la importancia de entender las percepciones junto con las motivaciones y las prácticas de participación a la hora de consumir contenido, esto con el fin de obtener una comprensión integral de las audiencias y explicar el contexto del consumo y la forma y los procesos a través de los cuáles éste se produce (González-Bernal, 2016, p. 802). En ese mismo sentido Davis Mersey, Calder y Malthouse (2010) lo definieron como la colección de experiencias que los consumidores tienen con determinado producto o contenido, poniendo en relieve la manera en la que ese contenido o producto encaja en sus vidas. 
"Cuando las personas se involucran con una marca o producto de medios de comunicación, lo hacen porque están viviendo una experiencia determinada con ese producto o debido a él; por tanto, la marca está jugando un papel integral en la vida de esa persona. Así es como es indispensable para las marcas conocer las razones por las que se propician esas experiencias" (Davis Mersey, Calder y Malthouse, 2010, p. 41).

El engagement con medios audiovisuales implica inmersión y atención, tiempo dedicado, exposición a un contenido, variedad de conexiones emocionales y motivos, las cuáles se materializan en un conjunto de experiencias que una persona tiene al consumir un producto de comunicación (Napoli, 2010; Peck \& Malthouse, 2011; Perälä \& Helle, 2014).

Para Davis Mersey, Calder y Malthouse (2010) estas experiencias de los usuarios tienen una dimensión personal y una social-interactiva. La personal hace referencia al conjunto de manifestaciones que las personas suelen tener con medios tradicionales; por otro lado, la parte social-interactiva es más específica para el consumo de medios en internet, e incluso este conjunto de experiencias se comparten con lo que un usuario experimenta consumiendo redes sociales. En general, a través del consumo de medios de comunicación, no importa cuál sea su formato, las personas buscamos estimulación, inspiración, interacción con otras personas a propósito del contenido, afirmación de autoestima y sensaciones intrínsecas de gozo o satisfacción. En las experiencias de engagement social-interactivo siempre están incluidas las personales. En complementariedad, el compromiso social-interactivo está motivado por la relevancia social y una experiencia de compromiso y de comunidad mucho más amplia, por eso se valoran en estas dimensiones aspectos relativos a la participación con otros y la socialización. El sentido de utilidad y de gozo intrínseco también están presentes en estas experiencias de implicación.

Las dimensiones planteadas por dichos autores comprenden: experiencias de estimulación e inspiración, de facilitación social, de temporalidad, de autoestima y conciencia cívica, de entretenimiento intrínseco, utilidad, participación y socialización y sentido de comunidad. De acuerdo a este modelo, algunas dimensiones comprenden experiencias de engagement individual y otras experiencias sociales e interactivas (ver figura 1). A partir de 37 experiencias concretas se genera el cuestionario de medición seleccionado para este trabajo de investigación.

Figura 1. Modelo de engagement

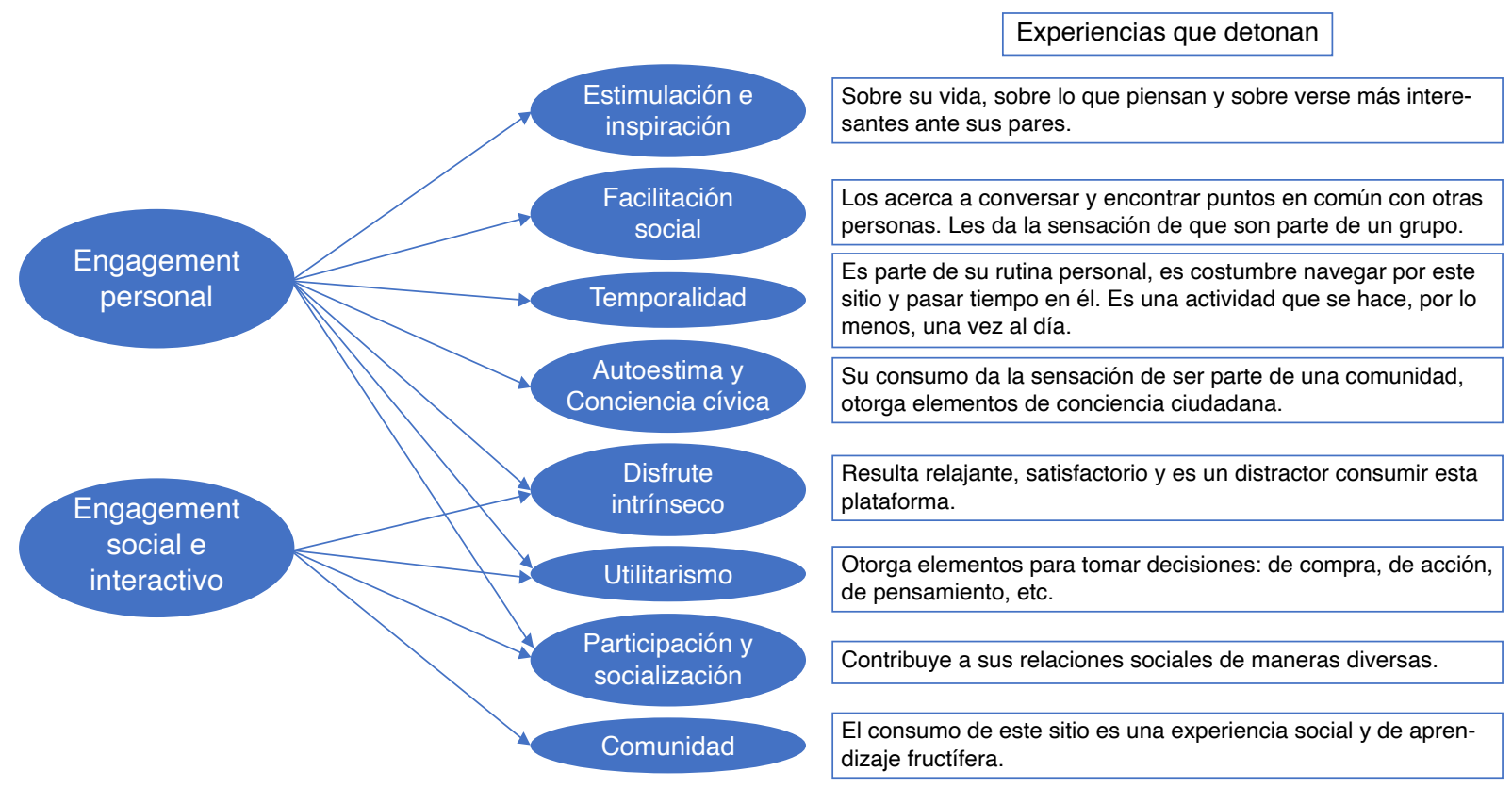

Fuente: Elaboración a partir del modelo de Davis Mersey, Malthouse y Calder, 2010 
En este trabajo se tomó este modelo que tiene sus antecedentes en la perspectiva de usos y gratificaciones de Katz \& Gurevitch (1973) y McQuail (1983) y luego adaptada por Ruggiero (2000), para conocer las experiencias que los millennials mexicanos tienen al usar Netflix. Así se pretende que a través de este análisis tener una medida del engagement en el momento del consumo, pero también la forma y los procesos que suceden a partir de ese consumo. La selección de este modelo se fundamenta en su utilización sostenida como referencia de análisis de experiencia de engagement con medios digitales y que ha sido probada y validada en diferentes estudios (Mersey, Malthouse y Calder, 2012; Isaac y Malthouse, 2016; Guo, 2018; Lin, Chen y Sung, 2018; Sung, Kang y Lee, 2018)

\section{Método}

En este estudio se parte del cuestionamiento qué tipos de experiencias de consumo se satisfacen con Netflix. El propósito el conocer el engagement con la plataforma de streaming Netflix y, a partir de lo anterior:

a) Conocer con mayor profundidad creencias, pensamientos e ideas que los sujetos de investigación tienen sobre sus experiencias de engagement con esta plataforma conceptualizadas a partir del modelo elegido.

b) Conocer la pertinencia del engagement para medir el consumo de plataformas audiovisuales como Netflix.

c) Encontrar arquetipos de usuarios de Netflix, a partir de las experiencias de consumo más significativas.

Para describir las experiencias de engagement de millennials mexicanos con Netflix, se seleccionó un diseño cuantitativo. A partir de este diseño se buscó: a) utilizar una herramienta validada para medir experiencias de engagement con un medio digital como Netflix y, b) obtener, a partir de esa herramienta, más información sobre las diversas dimensiones en las que el consumo de Netflix impacta en sus vidas.

Ésta es una investigación empírica-analítica (Arnal, Del Rincón y Latorre, 1992) con un diseño descriptivo, no experimental y transeccional (Hernández-Sampieri, 2014), pues busca describir las experiencias del engagement entre los millennials de la Zona Metropolitana de Guadalajara en México. Es una investigación exploratoria y no experimental, porque no hay intención de manipular deliberadamente las variables a investigar, sino observar un fenómeno tal y como se lleva a cabo en su entorno natural. En este sentido no hay hipótesis asociadas al estudio, pues la intención es tener un primer acercamiento a esta forma de mapear experiencias de engagement.

Asimismo, se trata de una investigación transeccional porque se recolectaron datos en un momento único y se describirán las experiencias analizadas. El instrumento cuantitativo seleccionado para la recolección de datos es un cuestionario electrónico y auto administrado, con preguntas cerradas de respuesta única y escalamiento estimativo tipo Likert. Los ítems se originaron a partir del modelo de Davis Mersey, Malthouse y Calder (2010), contextualizados al uso de Netflix con una escala estimativa en la que los usuarios pudieran señalar el nivel de acuerdo o desacuerdo que tienen sobre las 37 experiencias de engagement identificadas en el mismo. También se añadieron otras preguntas que permitieran tener información descriptiva sobre la muestra seleccionada. La escala seleccionada fue del 1 al 7, donde 1 era "muy en desacuerdo" y 7 "muy de acuerdo", más la opción 8 de "no aplica" en el caso de que la percepción sobre la presencia de esa experiencia de engagement con Netflix fuera nula. Este rango de 7 entre la respuesta más afirmativa y la negativa, se hizo para lograr un mayor nivel de discriminación de opciones y la tener resultados más altos de fiabilidad de los constructos analizados (González y Pazmiño, 2015). 
El universo de este estudio fueron millennials de la Zona Metropolitana de Guadalajara nacidos entre el año 1981 y el año 2000, dicha población es de $\mathrm{N}=1,347,028$ de acuerdo al IIEG (2018). En México las personas en este rango de edad son quienes más acceso a internet tienen, más tiempo de consumo presentan y los que definen con mayor disposición de pago de servicios por suscripción por internet (IFT, 2019).

La muestra fue intencionada; se utilizó una probabilidad de acierto (p) de 0.5 para determinar el tamaño de la muestra, con un nivel de confianza (z) de $95 \%=1.96 \%$ y un margen de error $€$ de 5\%. Lo anterior dio por resultado una muestra (n) de 384 jóvenes; se realizó un muestreo por conveniencia y se logró llegar a una muestra final de 574 encuestas, lo cual bajo el margen de error (e) a $4.09 \%$. Aunque el cuestionario fue contestado por 762, después de tomar decisiones respecto al alcance de la muestra, se tuvieron que invalidar algunos de ellos, otros fueron excluidos porque no cumplir con los filtros (respondieron estando en otra ciudad o no tenían la edad indicada) o simplemente no completaron la totalidad de preguntas de la encuesta.

Para obtener acceso a la muestra por conveniencia, se seleccionó el público universitario de licenciatura y posgrados, porque entre las personas de las edades seleccionadas, son los que presentan un promedio más alto de disponibilidad tecnológica. Se envió la encuesta a través de correos electrónicos de los alumnos de algunas de las principales universidades de la Zona Metropolitana de Guadalajara, se contactó a profesores y se visitó físicamente las universidades para asegurar la calidad de la aplicación y tener un margen muestral más amplio. Se invitó a participar a las personas en salones de clase con el permiso de los profesores. El cuestionario fue autoadministrado en la plataforma de Questionpro en presencia del investigador y los participantes accedieron a responderlo firmando un consentimiento.

Posterior a la recolección de la información, la plataforma de QuestionPro proporcionó una base de datos en Excel, se revisó la codificación y se limpiaron los datos, después se transfirieron al programa de análisis estadístico SPSS para ser procesados. Se corrieron diversos análisis descriptivos, así como la relación entre algunas variables, sobre todo relacionada con el consumo de las plataformas elegidas: los tiempos de consumo, las diferencias en edades y sexo respecto al consumo y las formas de engagement encontradas de acuerdo al modelo de Davis Mersey, Malthouse y Calder (2010).

Los 574 participantes viven en alguno de los 5 municipios pertenecientes a la Zona Metropolitana de Guadalajara: Guadalajara, Zapopan, Tonalá, Tlaquepaque y Tlajomulco y otros municipios aledaños más pequeños. En la ZMG, los municipios con mayor número de habitantes son Guadalajara y Zapopan, con 1.53 y 1.36 millones de habitantes (IIEG, 2018) respectivamente.

Respecto a los demás demográficos, la encuesta fue respondida por 365 mujeres y 209 hombres, correspondiendo al $63.6 \%$ y al $36.4 \%$, respectivamente. Esta distribución es relativamente consistente con el hecho de que, de acuerdo al IIEG (2018), en la zona metropolitana de Guadalajara, hay una mayor población de mujeres que de hombres, particularmente en el municipio de Guadalajara. También tiene que ver con la población estudiantil a la que se cuestionó, que fueron de áreas de ciencias sociales y de empresariales y ciencias económicas, cuya población femenina es mayor a la masculina.

La distribución de la muestra quedó más representada por personas entre los 18 y los 23 años, no obstante, hay representación de participantes de todas las edades del rango seleccionado. Este porcentaje influirá en los demás resultados, que serán más representativos para algunas franjas de edad. Este resultado se obtuvo por la recepción que tuvo la respuesta al cuestionario entre población universitaria, así como la facilidad de llegar a ese clúster. Entre la muestra, el 56.3\% ha terminado el bachillerato, el $34.7 \%$ ha terminado una licenciatura, mientras que el $9.1 \%$ cuenta con algún posgrado. $77.2 \%$ de la muestra está en el rango de los $18-26$ años y $22.8 \%$ entre los 27 y 37 años. 


\section{Resultados}

Para responder a los objetivos planteados, se partió corroborando el uso de Netflix. La penetración de la plataforma, se pudo confirmar la pertinencia de la muestra en la utilización de esta OTT, pues el $99.8 \%$ de la muestra seleccionada la utiliza, por tanto, se corrobora también la disposición del mercado mexicano a pagar una plataforma de streaming. Otro dato que resulta interesante, por la identificación de uso de pantallas entre la población joven y por resultar un elemento unido a la experiencia de consumo es cómo se accede a Netflix. Entre la muestra, 15\% lo hace a través de su celular, $28.9 \%$ a través de una computadora portátil, $6.6 \%$ en una tableta y $50.3 \%$ en una televisión inteligente. El porcentaje que resta $(2.6 \%)$ no consume esta plataforma de streaming. Además de lo anterior, se solicitó información sobre el tiempo de consumo de Netflix entre semana y en fin de semana (ver Tabla 1).

Tabla 1. Tiempo de consumo de Netflix entre semana y en fin de semana

\begin{tabular}{|l|c|c|c|c|c|}
\hline \multirow{2}{*}{ Pregunta } & Sexo & N & Media & $\begin{array}{c}\text { Desviación } \\
\text { Estándar }\end{array}$ & $\begin{array}{c}\text { Media de error } \\
\text { estándar }\end{array}$ \\
\hline \multirow{2}{*}{ Min. al día que se pasa en Netflix } & $\mathrm{M}$ & 361 & 193 & 457.72 & 24.09 \\
\cline { 2 - 6 } & $\mathrm{H}$ & 207 & 136.90 & 167.29 & 11.62 \\
\hline $\begin{array}{l}\text { Min. al día que se pasa en Netflix en } \\
\text { fin de semana }\end{array}$ & $\mathrm{M}$ & 364 & 268.56 & 867.71 & 45.48 \\
\cline { 2 - 6 } & $\mathrm{H}$ & 207 & 156.41 & 138.13 & 9.60 \\
\hline
\end{tabular}

Fuente: Elaboración propia

De acuerdo a la información extraída, los jóvenes mexicanos dedican tiempo importante de su semana al consumo de ésta y las mujeres presentan un consumo de tiempo más importante que los hombres. En lo relativo a las experiencias, se determinó aplicar los siguientes análisis: alfa de Cronbach, análisis descriptivos y análisis factorial exploratorio. Se utilizó este tipo de análisis pues dado que los constructos estudiados para cada plataforma son muchos, era necesario buscar la mayor síntesis y agrupación de los componentes principales, para analizarla con mayor pertinencia.

Para descubrir las experiencias de los millennials mexicanos con Netflix, el análisis factorial exploratorio sirvió para minimizar los atributos de engagement presentes en su consumo. Este tipo de análisis permitió descubrir experiencias de uso más significativo, tomando en cuenta que se considera una variable de correlación alta aquella que está muy cercana o por encima del 0.6. Este análisis también ayuda a confirmar la validez de los constructos en relación a lo que estamos midiendo: dimensiones de engagement con Netflix.

La prueba de alfa de Cronbach permitió determinar una consistencia interna alta de cada uno de los constructos analizados. En este sentido, los resultados nos permiten determinar que las experiencias medidas para Netflix nos arrojan resultados confiables, pues se ubican entre el (.946). Es decir, el modelo de Davis Mersey, Calder y Malthouse es útil para medir la experiencia de engagement de los usuarios. Posterior al análisis de fiabilidad, se hicieron dos tipos de análisis, uno descriptivo, para conocer las medianas y la desviación estándar de los atributos de engagement. Para representar estos resultados se tomó la decisión de solo elegir aquellos que tenían una mediana por arriba de 5.00 (ver Tabla 2), tomando en cuenta que la escala fue del 1 al 7; y para los atributos más bajos por plataforma fueron elegidos los que presentaron una mediana por debajo de 2 . 
Tabla 2. Atributos más altos de engagement en Netflix

\begin{tabular}{|l|c|c|}
\hline Atributo & Mediana & $\begin{array}{l}\text { Desviación } \\
\text { estándar }\end{array}$ \\
\hline El contenido de Netflix me estimula a pensar sobre diferentes temas & 5.00 & 1.708 \\
\hline El uso de Netflix me da pie a socializar sobre diferentes temas. & 5.00 & 1.845 \\
\hline Ver Netflix me relaja bastante & 5.00 & 1.876 \\
\hline Me gusta ver Netflix cuando estoy tomando un descanso o comiendo & 5.00 & 2.002 \\
\hline Ver Netflix me produce satisfacción & 5.00 & 1.895 \\
\hline Uso Netflix como parte importante del contenido de entretenimiento que consumo & 5.00 & 1.984 \\
\hline $\begin{array}{l}\text { Me he descubierto hablando sobre Netflix o sobre contenido de Netflix en } \\
\text { conversaciones con otras personas }\end{array}$ & 6.00 & 1.900 \\
\hline El contenido de Netflix me ha dado temas de conversación & 6.00 & 1.824 \\
\hline Ver Netflix se ha convertido en parte de mi rutina & 5.00 & 2.120 \\
\hline
\end{tabular}

Fuente: elaboración propia

La percepción de los jóvenes indica la asociación de uso de esta plataforma como elemento de interacción con otras personas, con el disfrute, el descanso y de gratificación. La información demuestra que el uso de esta plataforma detona también vínculos relacionados con la inspiración y la socialización. Es decir, los usuarios piensan su consumo de Netflix como una alternativa clara de descanso y les sugiere emociones relacionadas con el gozo individual y social, en la medida que es compartido por sus pares; la audiencia de Netflix es también una audiencia social y multipantallas, que incorpora las redes sociales y la multitarea y como parte de su experiencia de consumo.

Tabla 3. Atributos más bajos de engagement con Netflix

\begin{tabular}{|l|c|c|}
\hline Atributo & Media & $\begin{array}{l}\text { Desviación } \\
\text { Estándar }\end{array}$ \\
\hline Una de las razones por las que me gusta Netflix es la socialización con otras personas & 2.00 & 1.671 \\
\hline $\begin{array}{l}\text { El contenido de Netflix me ha dado herramientas para manejar de mejor manera mi } \\
\text { dinero }\end{array}$ & 2.00 & 1.378 \\
\hline Me he descubierto dando algún consejo a partir del contenido que he visto en Netflix. & 2.00 & 1.702 \\
\hline El contenido de Netflix me ha provisto de información para tomar decisiones relevantes & 2.00 & 1.484 \\
\hline Netflix me ha provocado alguna decisión de compra & 2.00 & 1.567 \\
\hline Soy una mejor persona usando Netflix & 2.00 & 1.582 \\
\hline Usar Netflix me ha hecho sentir en alguna ocasión como un buen ciudadano & 2.00 & 1.538 \\
\hline Una de las primeras actividades del día es revisar Netflix & 2.00 & 1.495 \\
\hline
\end{tabular}

\section{Fuente: Elaboración propia}

Los jóvenes mexicanos no perciben su consumo de Netflix con experiencias relacionadas a una conducta posterior, como la compra, así mismo, no la distinguen como una red social y, en ese sentido, como una forma de relacionarse con su comunidad de una manera más significativa (ver Tabla 3), aunque en el análisis factorial y en la evidencia de los atributos más altos percibidos, sí se identifica que el consumir esta plataforma de SVOD les permite coincidir en temas de conversación con otras personas.

El análisis factorial permitió hacer una identificación más fina de las experiencias y gratificaciones encontradas en Netflix y las dimensiones a las que pertenece ese consumo. Las experiencias que presentan significatividad más alta son las relacionadas con el disfrute intrínseco y la sensación de 
comunidad. Dichos resultados explican que el consumo de esta plataforma se asocia sobre todo con experiencias de descanso y relajación y que el uso de esta plataforma y las decisiones de contenido están altamente influidas por otras personas, por su contexto y por la manera en la que se promueve la plataforma a través de redes sociales o, en general, lo que se dice de esta plataforma y de sus contenidos en sus círculos sociales. Consumir Netflix es una experiencia altamente social, sin que el ámbito personal no sea relevante. De acuerdo a los resultados, el consumo de esta OTT observa una diversidad de experiencias, de las que el modelo elegido puede dar cuenta (ver Figura 2), lo cual también describe lo multidimensionalidad de las motivaciones, las emociones y las creencias alrededor de su consumo.

Figura 2. Estimado de engagement con Netflix por dimensiones

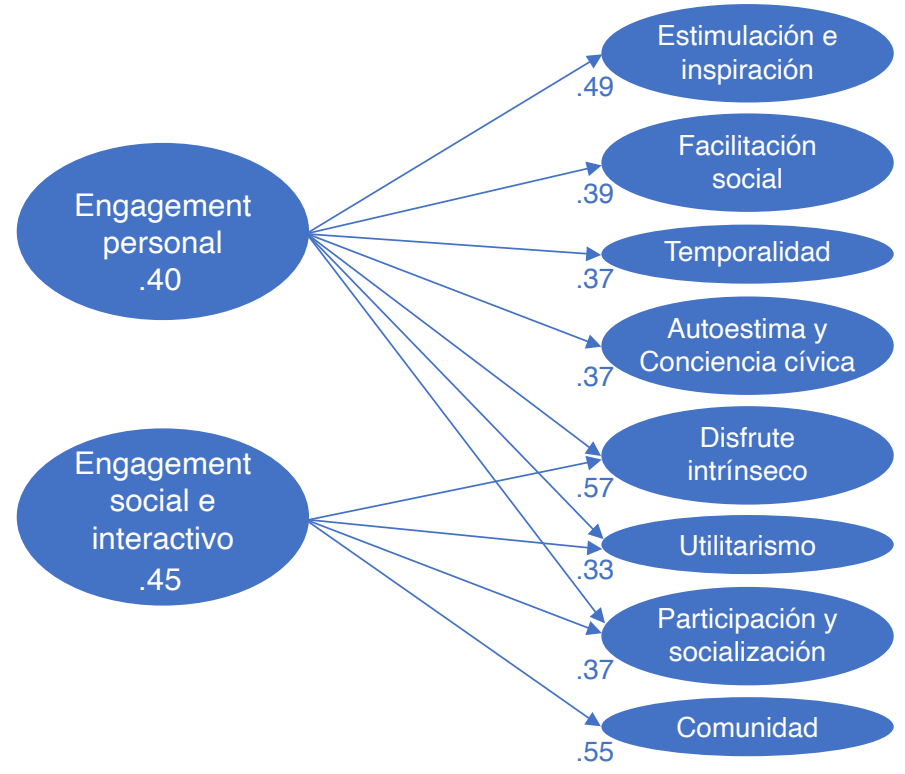

Fuente: Elaboración propia

Esta prueba estadística también permitió responder al propósito de la construcción de arquetipos de usuarios. Los arquetipos facilitan la comunicación efectiva y ayudan segmentar los públicos además, de diseñar herramientas para mejorar el tratamiento que se le da a cada uno de ellos (Cabrero, Winschiers-Theophilus \& Nocera, 2016). Al construir estos subgrupos se pueden diferenciar desde una perspectiva conductual, incluyendo emociones y estados psicológicos (Helkkula, Kowalkowski, \& Tronvoll, 2018) entregando información relevante a quiénes deben idear campañas o estrategias diferenciadoras (Altuwairiqi, Jiang \& Ali, 2019).

Este análisis logró responder al objetivo de diferenciar cuatro tipos de usuarios de Netflix, entre los jóvenes mexicanos. La categorización de estos usuarios se realizó con base en las experiencias de consumo que se organizan gracias al análisis factorial (ver Tabla 4). Éstas son: (1) relajado, que se conecta a la plataforma para descansar, desconectarse del trabajo o los estudios y repite esta acción de manera rutinaria. Ver Netflix es algo que hace constantemente en su tiempo libre. (2) reflexivo que luego de ver alguna serie, película o documental medita sobre lo visto, reflexiona y conecta con su experiencia y situaciones del entorno que lo rodea. (3) El social, que se conecta principalmente a la plataforma para establecer relaciones con otros (posteriormente en conversaciones), y generar interacciones, las que también le permiten conocer nuevas personas. Por último, (4) mejor persona que sienten que al usar la plataforma lo convierte en un mejor ciudadano apelando a su sentido de responsabilidad social. 
A partir de estos arquetipos, se corrobora la utilización de Netflix como una pantalla que conecta a los usuarios con experiencias de descanso y que les provoca sentimientos positivos y de gratificación inmediata, no obstante también se incorporan elementos de reflexión en su consumo, sobre todo relacionados con temáticas que ven en los contenidos que descubren en Netflix. Así mismo, se confirma que al utilizar Netflix también se conectan con otros y, a su vez, otros influyen los contenidos que eligen en la plataforma, en tanto estos contenidos suelen ser temas de conversación recurrentes en sus círculos sociales. Así mismo, esta OTT no es una plataforma que claramente tenga efectos colectivos que repercutan en la generación de capital social, aunque aparezcan dos experiencias relacionadas con elementos de participación ciudadana, la naturaleza de esta plataforma es primordialmente para entretener, aunque de manera esporádica, muy probablemente los usuarios descubren contenidos que puedan conectarlos con temas de implicación social o política.

Tabla 4. Análisis factorial sobre los atributos de engagement con Netflix

\begin{tabular}{|c|c|c|c|c|c|c|c|}
\hline \multirow{2}{*}{ Atributo } & \multicolumn{7}{|c|}{ Componente } \\
\hline & 1 & 2 & 3 & 4 & 5 & 6 & 7 \\
\hline \multicolumn{8}{|l|}{ Arquetipo de usuario "relajado" } \\
\hline $\begin{array}{l}\text { Uso Netflix como parte importante del contenido de } \\
\text { entretenimiento que consumo }\end{array}$ & 0.788 & & & & & & \\
\hline Ver Netflix me relaja bastante & 0.694 & & & & & & \\
\hline El contenido de Netflix me ha dado temas de conversación & 0.694 & & & & & & \\
\hline Ver Netflix se ha convertido en parte de mi rutina & 0.691 & & & & & & \\
\hline $\begin{array}{l}\text { Me he descubierto hablando sobre Netflix o sobre } \\
\text { contenido de Netflix en conversaciones con otras personas }\end{array}$ & 0.664 & & & & & & \\
\hline Ver Netflix me provoca satisfacción & 0.640 & & & & & & \\
\hline $\begin{array}{l}\text { Me gusta ver Netflix cuando estoy tomando un descanso o } \\
\text { comiendo }\end{array}$ & 0.637 & & & & & & \\
\hline $\begin{array}{l}\text { Ver Netflix mejora mi estado anímico, me hace sentir más } \\
\text { contento }\end{array}$ & 0.624 & & & & & & \\
\hline \multicolumn{8}{|l|}{ Arquetipo de usuario "reflexivo" } \\
\hline El contenido de Netflix me hace pensar de nuevas maneras & & 0.769 & & & & & \\
\hline $\begin{array}{l}\text { El contenido que he visto en Netflix me ha hecho } \\
\text { reflexionar con profundidad }\end{array}$ & & 0.737 & & & & & \\
\hline $\begin{array}{l}\text { El contenido de Netflix me estimula a pensar sobre } \\
\text { diferentes temas }\end{array}$ & & 0.714 & & & & & \\
\hline El contenido de Netflix inspira aspectos de mi propia vida & & 0.651 & & & & & \\
\hline \multicolumn{8}{|l|}{ Arquetipo de usuario "social" } \\
\hline $\begin{array}{l}\text { Una de las razones por las que me gusta de Netflix es la } \\
\text { socialización con otras personas }\end{array}$ & & & 0.704 & & & & \\
\hline $\begin{array}{l}\text { Netflix facilita que los usuarios del mismo interactuemos y } \\
\text { demos retroalimentación sobre su contenido }\end{array}$ & & & 0.700 & & & & \\
\hline Me gusta conocer personas que también ven Netflix & & & 0.648 & & & & \\
\hline $\begin{array}{l}\text { Usar Netflix me ha hecho sentir en alguna ocasión como un } \\
\text { buen ciudadano }\end{array}$ & & & & 0.737 & & & \\
\hline \multicolumn{8}{|l|}{ Arquetipo de usuario "mejor persona" } \\
\hline $\begin{array}{l}\text { Una de las primeras actividades del día que hago es } \\
\text { revisar Netflix }\end{array}$ & & & & 0.727 & & & \\
\hline Soy una mejor persona usando Netflix & & & & 0.687 & & & \\
\hline
\end{tabular}

Fuente: Elaboración propia 


\section{Discusión y conclusiones}

Las audiencias se conectan de diferentes maneras con los medios y los contenidos con un mayor control sobre la forma en la lo consumen. Este estudio se aproxima a entender las formas específicas en las que se da un consumo, en el que queda demostrado que es activo, y cada vez es mayor el tiempo que destinan a su uso. Por otra parte, ese mayor uso, en algunos usuarios, genera la sensación de pasar mucho tiempo en Netflix y sentir culpa por eso. De acuerdo a los datos encontrados, los jóvenes pasan un promedio 4.4 horas. en fines de semana y 2.2 horas. entre semana, usándola; así mismo, el atributo de: "sentir a veces culpa por pasar tanto tiempo en Netflix" obtuvo un valor muy alto en el análisis factorial de datos (0.797). Estos puntos sustentan la penetración de uso y tiempo de esta $S V O D$. Gracias a los análisis factoriales, fue posible identificar aspectos muy propios sobre el engagement y confirmar que el modelo de Davis Mersey, Calder y Malthouse sirve para ayudar a esta identificación de cualidades de conexión con medios digitales, como en este caso uno de contenidos audiovisuales por suscripción.

A partir de los resultados, se puede distinguir una habituación considerable a esta forma de consumo, a estos modelos de negocio y a lo que esta forma de visionado le aporta al consumidor $m i$ llennial mexicano. Además, se puede concluir una penetración positiva de la marca en el mercado mexicano, tomando en cuenta que ya hay una competencia mayor de servicios similares, conectando además esto con la discusión bibliográfica en la que hay indicios de que la fragmentación ha facilitado la aceptación de modelos de negocio por suscripción.

Entre la muestra, la televisión inteligente se empieza a imponer como un dispositivo que tiene presencia significativa en casa y que se está usando como fuente de entretenimiento. Esta información confirma datos de Statista (2016) que señalan que las actividades más realizadas en Smart TV por los mexicanos son ver películas, series y videos, seguida por escuchar música. Este dato está en consonancia con información de INEGI (2019) en el que se encontró que, en el 2019, el 58.7\% de los hogares mexicanos reciben señal de internet a través de una televisión inteligente. No obstante, el celular es un dispositivo práctico para ver contenido para visionado de esta plataforma, pero se puede deducir que se usa como una segunda pantalla.

En el caso específico de Netflix, en esta investigación sobresalen claramente las experiencias de estimulación e inspiración, temporalidad y la facilitación social, relacionadas ambas con atributos de engagement personal y solo la de participación y socialización en el caso del ámbito social. Dichas dimensiones hacen alusión además al fácil acceso a una plataforma y a la influencia del entorno para usar o ver contenido en dicha plataforma y al fenómeno de la televisión social, en la que el consumo de otros, influye el consumo personal (Shin, 2013); lo cual se demuestra en esta investigación.

A partir de la aplicación de este modelo se logra distinguir que el público se relaciona de manera particular con la marca Netflix, y estos usuarios experimentan motivaciones diferenciadas y variadas al usar la plataforma. Tomando en cuenta que el modelo de engagement elegido describe una colección más vasta de experiencias, los resultados de esta investigación permiten identificar experiencias particulares para Netflix, la forma en la que el consumo de esta plataforma conecta a los jóvenes con otros aspectos de su vida, y la forma en la que el consumo de plataformas digitales de suscripción se ha convertido en una parte importante de sus usos y costumbres de ocio. De igual manera, se detectan cuatro tipos de usuarios de Netflix mexicanos, que combinan experiencias individuales y sociales, relacionadas primordialmente con la relajación.

Este análisis permite pues responder a la pregunta de investigación, logrando identificar tipos de experiencias de consumo con Netflix. Así mismo se encontró una dedicación de tiempo relevante al consumo de contenido digital, la incorporación de rutinas a sus actividades diarias, la socializa- 
ción de contenidos que están viendo en plataformas como temas de conversación con sus pares y la detonación de su interés por determinados temas. La edad es un factor relevante para el tipo de consumo, para el pago por plataformas, en donde el $96.7 \%$ de la muestra paga por un servicio mensual de manera propia o bien, por sus familias; $y$, de nuevo, para el tiempo que se consume, según los datos obtenidos en esta encuesta, explicados ya con antelación.

Por otro lado, se percibe a través de este estudio que hay una conciencia más explícita sobre el engagement que se tiene con el consumo en relación a una plataforma específica. Queda demostrado que los más jóvenes dentro del universo estudiado tienen un mayor engagement y éste se manifiesta de diversas maneras: les es común pasar tiempo consumiendo contenido en Netflix, están habituados a que este sitio es un tema de conversación con sus pares y su disposición a gastar dinero en este tipo de oferta, es positiva, pues les genera algún tipo de valor a partir de las experiencias de engagement que ya han sido explicadas.

Otro hallazgo importante de esta investigación, es que es posible utilizar modelos de engagement para reconocer el vínculo de las personas con las plataformas y los contenidos que consumen. Autores como Bergillos (2017) consideran que respecto al consumo digital se prevé que las audiencias tenderán a ser más conscientes y conocedoras de su forma de consumo y que reconozcan a través de ello, que les gusta algún contenido y que, por tanto, merece su tiempo y atención. Sin embargo, es necesario complementar este consumo con otros elementos que ayuden a comprender mejor por qué las personas deciden consumirlo. Para que el público pueda comunicar una mayor conciencia respecto a lo que ve, probablemente, sea necesario puntualizar en una segunda etapa el tipo de consumo en contextos más concretos y, probablemente, a partir de contenidos, y no sólo plataformas.

En esa línea se podría explorar perspectivas de personas en la industria (directivos, productores, creativos), una muestra específica de contenidos de la plataforma, y profundizar en la percepción de las audiencias a través de una metodología cualitativa, de tal manera que se pueda poner de relevancia aspectos particulares que pueden ser generadores de engagement, asumiendo que a través de esto, los usuarios serán capaces de orientar mejor el carácter de su vinculación con contenidos y den cuenta de las decisiones que orientan su consumo de productos específicos.

Estudiar el engagement depende de diversos factores, que son influidos por los modos y el tiempo de consumo, en los cuáles las plataformas son solo un factor asociado. Hasta el momento, con este estudio, sabemos que Netflix es muy utilizado por los jóvenes mexicanos y que éste otorga experiencias de disfrute personal variadas que incluso son de valor en la relación con sus pares o en la exposición de ideas que tienen sobre la vida o sobre determinado tipo de información.

Los jóvenes son un público consumidor de plataformas digitales, adaptados a los efectos de la convergencia y con disposición de combinar esas posibilidades con las opciones que les dan las redes sociales. Parte de su consumo está dado por estar conectados con los demás y eso detona sentimientos, pensamientos y comportamientos de los que manifiestan ser conscientes. Esto sugiere que el público tiene autodeterminación sobre su consumo y que es necesario adoptar concepciones más integrales sobre las audiencias, que no despersonalicen al público y que se comprenda desde la industria que se otorga un servicio a personas concretas, que tienen necesidades y en la medida que se les conozca mejor se les puede atraer y retener. Quienes logren generar esa diferenciación y ventaja competitiva tendrán mayor posibilidad de ser exitosos en una industria que tiene una competencia brutal por quedarse con el tiempo libre de las personas (Rajala \& Korhonen, 2020). 


\section{Bibliografía}

Armendáriz, J. (2019). Netflix invertirá 200 MDD para producciones mexicanas en el 2020. https://www.informador.mx/economia/Netflix-invertira-200-MDD-para-producciones-mexicanasen-2020-20190906-0073.html

Arnal, J., Del Rincón, D. \& Latorre, A. (1992). Investigación educativa. Barcelona: Labor.

Asociación de Internet en México (2019). 15. Estudio sobre hábitos de los usuarios de Internet en México. https://bit.ly/3oScclQ

Barry, B. \& Crant, J. M. (2000). Dyadic communication relationships in organizations: An attribution/expectancy approach. Organization Science, 11(6), 648-664. https://doi.org/10.1287/ orsc.11.6.648.12537

Barthel, M. (2019). 5 key takeaways about the state of the news media in 2018, Pew Research Center. https://www.pewresearch.org/fact-tank/2019/07/23/key-takeaways-state-of-the-newsmedia-2018/

Belair-Gagnon, V., Nelson, J. L. \& Lewis, S. C. (2019). Audience engagement, reciprocity, and the pursuit of community connectedness in public media journalism. Journalism Practice, 13(5), 558-575.

Bergillos, I. (2017). ¿Dos caras de la misma moneda?: una reflexión sobre la relación entre engagement y participación en medios. Comunicación y Hombre, (14), 121-136.

Bowden, J. L. H. (2009). The process of customer engagement: A conceptual framework. Journal of Marketing Theory and Practice, 17(1), 63-74. https://doi.org/10.2753/MTP1069- 6679170105

Brodie, R. J., Ilić, A., Jurić, B. \& Hollebeek, L. (2013). Consumer engagement in a virtual brand community: An exploratory analysis. Journal of Business Research, 66(1), 105-114. https://doi. org/10.1016/j. jbusres.2011.07.029

Calder, B. J., Malthouse, E. C. \& Schaedel, U. (2009). An experimental study of the relationship between online engagement and advertising effectiveness. Journal of Interactive Marketing, 23 (4), 321-331. https://doi.org/10.1016/j.intmar.2009.07.002

Calder, B. J. (2010). Media concepts. In A. Peck \& E. C. Malthouse (Eds.), Medill on Media Engagement. New York: Hampton Press.

Calder, B., Isaac, M. S., \& Malthouse, E. (2016). How to capture consumer experiences: A context- specific approach to measuring engagement. Journal of Advertising Research, 56(1), 1-14. https://doi.org/10.2501/JAR-2015-028

Chan-Olmsted, S. M. \& Wolter, L. C. (2018). Perceptions and practices of media engagement: A global perspective. International journal on media management, 20(1), 1-24.

Davis Mersey, R., Malthouse E. \&. Calder B. (2010) Engagement with Online Media, Journal of Media Business Studies, 7:2, 39-56. https://doi.org/10.1080/16522354.2010.11073506

Davis Mersey, R., Malthouse, E. C. \& Calder, B. J. (2012). Focusing on the reader: Engagement trumps satisfaction. Journalism \& Mass Communication Quarterly, 89(4), 695-709.

De la Gala, M. Á. (2016). De la planificación en TV a la planificación audiovisual y social:desafios para la industria ante un cambio de paradigma en la medición de la eficacia publicitaria. Doctoral dissertation. Universidad Complutense de Madrid). 
Denktaş-Şakar, G., \& Sürücü, E. (2018). Stakeholder engagement via social media: an analysis of third-party logistics companies. The Service Industries Journal, 1-24.

Eizaguirre, L. y Peñafiel C. (2013) La utilización de las redes sociales como nuevas herramientas aplicadas al análisis de audiencia. Trípodos, $\mathrm{n}^{\circ}$ e33, pp. 157-172.

Ferrer-Conill, R., and E. C. Tandoc Jr. (2018). The Audience-Oriented Editor. Digital Journalism 6: 436- 453. https://doi.org/10.1080/21670811.2018.1440972.

Ferrucci, P. (2017). Technology allows audience role in news construction. Newspaper Research Journal, 38(1), 79-89.

García, J., Salaverría, R. y Masip, P. (2008). Convergencia Periodística en los Medios de Comunicación. Propuesta de Definición Conceptual y Operativa, I Congreso de la Asociación Española de Investigadores en Comunicación, Santiago de Compostela. http://dspace.si.unav.es/ dspace/bitstream/10171/5099/1/Ramon_Salaverria.pdf

González-Bernal, M. I. (2016). Hacia la comprensión del engagement de las audiencias de televisión, modelo conceptual multidimensional desde la comunicación. Palabra Clave 19(3), 769-809. https://doi.org/10.5294/pacla.2016.19.3.5

González, J., \& Pazmiño Santacruz, M. (2015). Cálculo e interpretación del Alfa de Cronbach para el caso de validación de la consistencia interna de un cuestionario, con dos posibles escalas tipo Likert. Revista publicando, 2(1), 62-67.

Gutiérrez-Rentería, M. E. (2014). Estrategias de grupo Televisa: del monopolio a la competencia: Análisis económico, político y social de la industria audiovisual en México. Editorial Académica Española.

Gutiérrez-Rentería, M. E. \& Hernández, C. E. L. (2014). La convergencia digital propicia convergencia entre industrias: principales empresas que participan en el mercado de capitales, concentran los mayores ingresos en 2013. Revista de comunicación (13), 144-162.

Gulyas, A., O’Hara, S. \& Eilenberg, J. (2019). Experiencing local news online: audience practices and perceptions. Journalism Studies, 20(13), 1846-1863.

Guo, M. \& Chan-Olmsted, S. M. (2015). Predictors of Social Television Viewing: How Perceived Program, Media, and Audience Characteristics Affect Social Engagement With Television Programming. Journal of Broadcasting \& Electronic Media, 59(2), 240-258. https://doi.org/10.10 80/08838151.2015.1029122

Harris, L., \& Dennis, C. (2011). Engaging customers on Facebook: Challenges for e-retailers. Journal of Consumer Behaviour, 10, 338-346. https://doi.org/10.1002/cb.375

Havens, T. \& Lotz, A. (2017). Understanding media industries. Oxford University Press

Hermans, L. \& Prins, T. (2020). Interest matters: The effects of constructive news reporting on Millennials' emotions and engagement. Journalism, https://doi.org/10.1177/1464884920944741

Hernández, M. \& Aguilar, R. (25 de enero de 2021). Netflix invertirá más de 25 millones de dólares en México para el 2021. Forbes. https://www.forbes.com.mx/exclusiva-netflix-invertiramas-de-300-millones-de-dolares-en-mexico-para-2021/

Hernández Sampieri, R., Fernández Collado, C. \& Baptista Lucio, P. (2014). Metodología de la investigación (Vol. 3). México: McGraw-Hill. 
Higgins, E. T. \& Scholer, A. (2009). Engaging the consumer: The science and art of the value creation process. Journal of Consumer Psychology, 19(2), 100-114. https://doi.org/10.1016/j. jcps.2009.02.002

Hollebeek, L. D. (2011). Demystifying customer brand engagement: Exploring the loyalty nexus. Journal of Marketing Management, 27(7-8), 785-807. https://doi. org/10.1080/0267257X.2010.500132

IAB (2020). Estudio de consumo de medios y dispositivos entre internautas mexicanos. https://d335luupugsy2.cloudfront.net/cms/files/65507/1600450982ECMyD_2020_ CorteGeneralVP.pdf

IIEG (2018). Población de Jalisco 2018. https://bit.ly/33fiQLS

Instituto Federal de Telecomunicaciones IFT (2019). Oferta y demanda de OTTs de contenido audiovisual en México. http://www.ift.org.mx/sites/default/files/contenidogeneral/medios-ycontenidos-audiovisuales/ofertaydemandadeottsdecontenidosaudiovisualesenmexico2019.pdf

Instituto Nacional de Estadística y Geografía INEGI (2019). Encuesta Nacional sobre disponibilidad y uso de tecnologías de la información de los hogares 2019. https://www.inegi.org. $\mathrm{mx} /$ contenidos/saladeprensa/boletines/2020/OtrTemEcon/ENDUTIH_2019.pdf

Jenner, M. (2017). Binge-watching: Video-on-demand, quality TV and mainstreaming fandom. International Journal of Cultural Studies, 20(3), 304-320. https://doi. org/10.1177/1367877915606485

Jenkins, H. (2008). Convergence Culture. Where Old and New Media Collide. Nueva York, NY: New York University Press.

Johnston, K. A., \& Taylor, M. (Eds.). (2018). The handbook of communication engagement. John Wiley \& Sons.

Katz, E., Blumler, J. G. y Gurevitch, M. (1973). Uses and gratifications research. The Public Opinion Quarterly, 37(4), 509-523.

Lin, L. \& Parsons, T. D. (2018). Ecologically valid assessments of attention and learning engagement in media multitaskers. TechTrends, 62(5), 518-524.

Lin, J. S., Chen, K. J. \& Sung, Y. (2018). Understanding the nature, uses, and gratifications of social television: Implications for developing viewer engagement and network loyalty. Journal of Broadcasting \& Electronic Media, 62(1), 1-20.

Lotz. A. (2014). The Television Will Be Revolutionized, Second Edition. New York: NYU Press.

McQuail, D. (1983). Mass Communication Theory, an Introduction. London: Sage Publications.

Medina, M., Herrero, M. \& Urgellés, A. (2017). Current and Emerging Issues in the Audiovisual Industry. John Wiley \& Sons: New York.

Moe, H., Poell, T. \& van Dijck, J. (2016). Rearticulating audience engagement: Social media and television. Television \& new media, 17(2), 99-107.

Mollen, A. \& Wilson, H. (2010). Engagement, telepresence and interactivity in online consumer experience: Reconciling scholastic and managerial perspectives. Journal of Business Research, 63 (9-10), 919-925. doi:10.1016/j.jbusres.2009.05.014 
Napoli, P. (2010). Audience evolution: New Technologies and the Transformation of Media Audiences. New York: Columbia University Press.

Neilson, T. (2018). 'I don't engage': Online communication and social media use among New Zealand journalists. Journalism, 19(4), 536-552. https://doi.org/10.1177/1464884916667871

Neira, E. (2015). La otra pantalla. Editorial UOC.

Netflix Media Center (2017). 2017 on Netflix. A year in Bingeing. Consultado en: https://about. netflix.com/en/news/2017-on-netflix-a-year-in-bingeing

Nielsen Ibope (2020). Digital Consumer Survey México 2020. https://www.nielsenibope. com/2020/09/14/digital-consumer-survey-mexico-2020/

Newman, N., Fletcher, R., Kalogeropoulos, A., Levy, D. A. \& Nielsen, R. K. (2019). Digital news report 2019. Reuters Institute and University of Oxford.

Oeldorf-Hirsch, A. (2018). The role of engagement in learning from active and incidental news exposure on social media. Mass communication and society, 21(2), 225-247.

Orozco-Gómez, G. (1997). Medios, audiencias y mediaciones. Revista Científica de Comunicación y Educación, 4(8), 25-30. https://doi.org/10.3916/comunicar

Papí-Gálvez, N. \& Perlado, M. (2018). Investigación de audiencias en las sociedades digitales: su medición desde la publicidad. El profesional de la información, v. 27, n. 2, pp. 383-393.

Peck, A. \& Malthouse, E. C. (2011). Medill on media engagement. Creskill: Hampton Press, Inc

Perälä, R. y Helle, M. (2014). Media landscapes and media engagement. En G. Patriarche (ed.), Transforming audiences, transforming societies cost action conference (p. 35). Ljubljana: Cost Action. Recuperado de http://www.cost-transforming-audiences.eu/what-was-published.html

PriceWaterHouse PWH (2020). Perspectives from the Global Entertainment \& Media Outlook 2020-2024. https://www.pwc.com/gx/en/entertainment-media/outlook-2020/ perspectives.pdf

Rajala, J. \& Korhonen, A. (2020). Streaming wars: competitive dynamics in the online video streaming industry. (Master's Thesis). Jyäskylä University, Finland.

Ruggiero, T. E. (2000). Uses and gratifications theory in the 21 st century. Mass Communication \& Society, 3(1), 3-37.

Statista (2016). Internet activities carried out on Smart TV in México 2016. https://www.statista. com/statistics/728378/smart-tv-activities-mexico/

Statista (2020). Video Streaming (SVoD) in México. https://www.statista.com/outlook/206/116/ video-streaming--svod-/mexico

Statista (2020b). Number of video demand subscribers in Latin America 2019 -2025. https://www. statista.com/statistics/562214/number-subscription-video-on-demand-subscriptions-latam/

Shin, D. H. (2013). Defining sociability and social presence in Social TV. Computers in human behavior, 29(3), 939-947. https://doi.org/10.1016/j.chb.2012.07.006

Sung, Y. H., Kang, E. Y. \& Lee, W. N. (2018). Why do we indulge? Exploring motivations for binge watching. Journal of Broadcasting \& Electronic Media, 62(3), 408-426. 
Van Weezel, A. \& Benavides, C. (2013). How to engage the audience? A study on using Twitter to engage newspaper readers. Handbook of social media management (pp. 703-713). Springer, Berlin, Heidelberg.

Van Weezel, A. \& Benavides, C. (2010). Adoption of high definition television in Chile.

Conference paper presented at 9th World Media Economic and Management Conference, Bogota, Colombia. 